\title{
Ultrastructural Demonstration of a Gastric Monosynaptic Vagal Circuit in the Nucleus of the Solitary Tract in Rat
}

\author{
Linda Rinaman, ${ }^{1}$ J. Patrick Card, ${ }^{2}$ James S. Schwaber, ${ }^{2}$ and Richard R. Miselis ${ }^{1}$ \\ University of Pennsylvania, Institute of Neurological Sciences, Department of Animal Biology, Philadelphia, Pennsylvania \\ 19104, and "Medical Products Department, E.I. du Pont de Nemours and Co., Experimental Station, E400, Wilmington, \\ Delaware 19898
}

Previous light microscopic work in the rat has demonstrated that many dendrites of vagal gastric motoneurons extend beyond the cytoarchitectural boundaries of the dorsal motor nucleus of the vagus (DMV) into the nucleus of the solitary tract (NST), where they overlap with the central terminal field of vagal gastric primary sensory neurons (Shapiro and Miselis, 1985a). In the present ultrastructural study, the synaptic relationship of vagal afferent terminals and vagal motor neurons and dendrites was examined. Following injection of cholera toxin-HRP conjugate (CT-HRP) into the dorsal and ventral musculature of the stomach wall, centrally transported tracer was visualized using tetramethylbenzidine or diaminobenzidine histochemistry. Light microscopy confirmed the presence of retrogradely labeled gastric DMV neurons whose dendrites extended into the overlying NST. The subnucleus gelatinosus of the dorsomedial NST at a level just rostral to the obex contained the highest density of vagal motoneuronal dendrites coextensive with transganglionically labeled vagal sensory afferents. Ultrastructural analysis of several NST subfields at this rostrocaudal level revealed many asymmetric synaptic contacts between labeled sensory afferents and labeled motoneuronal dendrites in the subnucleus gelatinosus but not elsewhere. Following CT-HRP injection into the cervical vagus nerve in separate animals, vagovagal synaptic contacts were again observed only in the subnucleus gelatinosus, despite extensive afferent labeling in other NST subnuclei. The demonstration of a monosynaptic gastric vagovagal circuit provides the anatomical substrate for the relay of gastric interoceptive information directly to gastric vagal motoneurons, which may play a unique role in the central regulation of gastric motility and secretion.

Visceral afferent signals modify visceral motor responses through peripheral and central neural relays (Burnstock et al., 1979; Novin et al., 1981; Powley and Laughton, 1981; Delbro and Gustafsson, 1986; Grundy et al., 1986; Gonella et al., 1987; Sawchenko et al., 1987). Vagal sensory modification of vagal motor activity provides the substrate for medullary "vagovagal" reflexes, which have traditionally been thought to involve at

\footnotetext{
Received June 10, 1988; revised Oct. 17, 1988; accepted Nov. 4, 1988.

This work was supported by an Office of Naval Research Fellowship (to L.R.) by NII I Grant GM 27739 (to R.R.M.), and by E.I. du Pont de Nemours and Co.

Correspondence should be addressed to Linda Rinaman, \% Dr. Richard R. Miselis, Animal Biology, 3800 Spruce Street, University of Pennsylvania, Philadelphia, PA 19104-6045.

Copyright (C) 1989 Society for Neuroscience $0270-6474 / 89 / 061985-12 \$ 02.00 / 0$
}

least one interneuron in the nucleus of the solitary tract (NST; Szentagothai, 1948; Norgren, 1978; Powley and Laughton, 1981; Ewart and Wingate, 1983). Although occasional monosynaptic contacts between vagal sensory neurons and vagal motoneurons have been reported in the dorsal motor nucleus of the vagus (DMV) in rat (Neuhuber and Sandoz, 1986), vagal sensory axons terminate with relative rarity in the DMV (Cottle, 1964; Norgren, 1978; Leslie et al., 1982a; McLean and Hopkins, 1985; Neuhuber and Sandoz, 1986). On the other hand, light microscopy has shown that a portion of the dendritic arbor of vagal gastric motoneurons extends beyond the classical cytoarchitectural boundaries of the DMV to enter the overlying NST, where a smaller subset of these dendrites overlaps extensively with the central terminal field of vagal gastric sensory neurons in the subnucleus gelatinosus of the dorsomedial NST (Shapiro and Miselis, 1985a). These anatomical observations support the potential for extensive monosynaptic, axodendritic contacts between vagal gastric sensory and motoneurons. The purpose of the present ultrastructural study was to characterize the relationship between gastric sensory and motor vagal profiles, in order to determine whether synaptic contacts occur between them.

\section{Materials and Methods}

Animals. Five adult male Sprague-Dawley rats (300-350 gm) were used in the combined light- and electron-microscopic (EM) analysis. Animals were maintained on a $12: 12 \mathrm{hr}$ photoperiod and had free access to food and water.

Injection procedures. Cholera toxin-HRP (CT-HRP; $0.25 \%$ as determined by Lowry assay) was prepared in our laboratory as described previously (Shapiro and Miselis, 1985b). Animals were anesthetized prior to surgery by intraperitoneal injection of Chloropent (Fort Dodge Labs, $3.2 \mathrm{ml} / \mathrm{kg}$ ). In 3 rats, 50-70 $\mu$ l CT-HRP was injected into the dorsal and ventral musculature of the stomach according to a procedure which, through extensive control experiments, has been found to effectively restrict the tracer to actual sites of injection (Rinaman and Miselis, 1987). In 2 separate animals, $5 \mu$ l CT-HRP was injected unilaterally into the cervical vagus nerve just below the nodose ganglion. These animals were included in order to increase the chance of observing potential vagovagal contacts in various subfields of the NST and/or in the DMV. In this procedure, the nerve was bluntly dissected from the carotid artery and gently held away from surrounding tissue. A $5 \mathrm{~mm}$ section of the exposed nerve was crushed and CT-HRP delivered within the nerve sheath at the crush site by multiple injections through a glass micropipette secured to the tip of a $10 \mu \mathrm{l}$ Hamilton syringe. Gentamicin $(0.25 \mathrm{ml}$, TechAmerica, $50 \mathrm{mg} / \mathrm{ml})$ was injected intramuscularly in all animals postoperatively as a prophylactic measure.

Histology. Seventy-two hours after stomach injection or $48 \mathrm{hr}$ following cervical nerve injection, animals were reanesthetized with Chloropent and killed by transcardiac perfusion fixation. The perfusatcs consisted of $75 \mathrm{ml}$ heparanized saline (400 units heparin/100 ml saline) 


\section{Stomach}
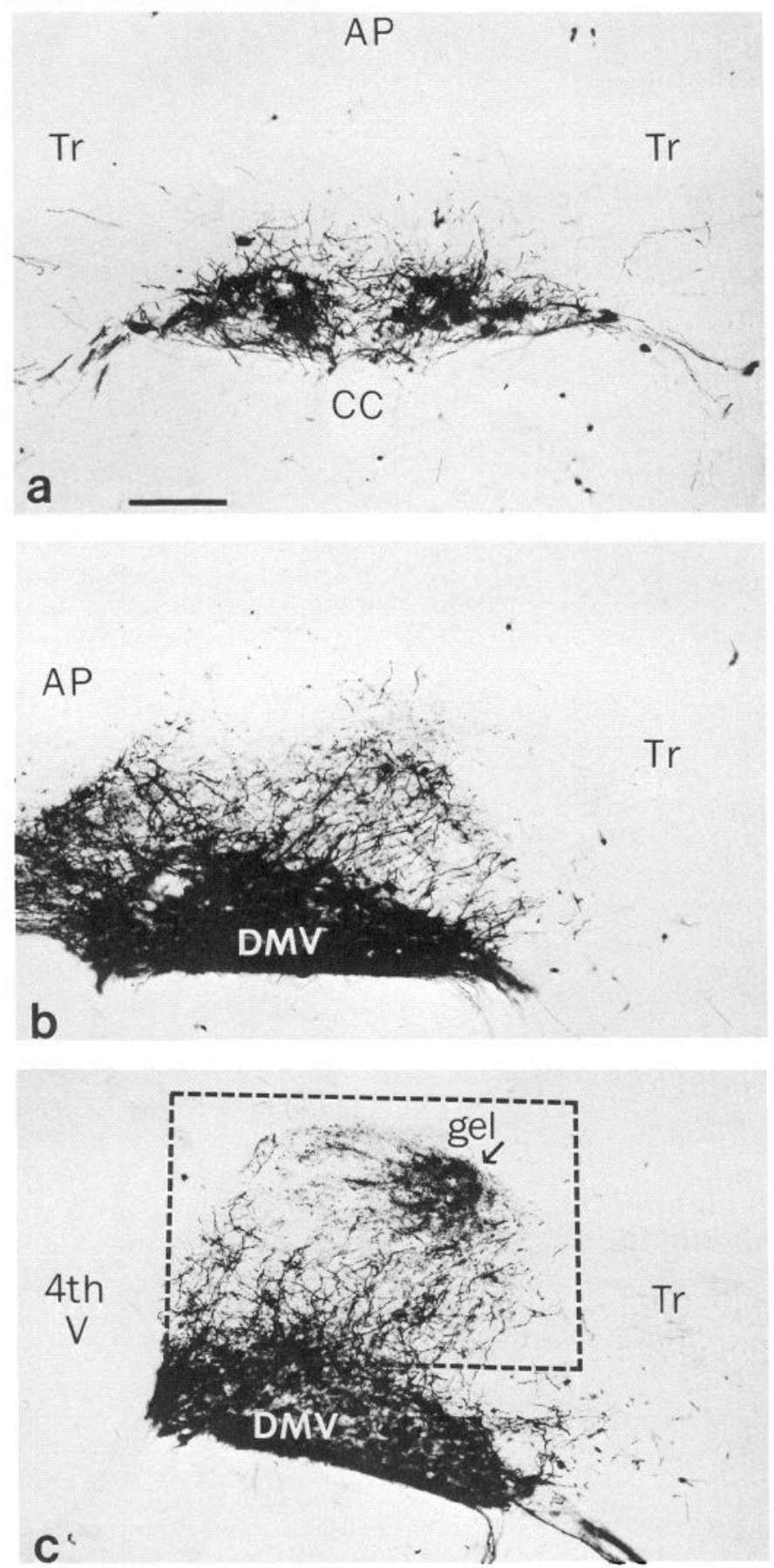

Figure 1. Light-field photomicrographs demonstrating labeling of motoneurons and sensory afferents in $(a)$ caudal, $(b)$ intermediate, and $(c)$ rostral levels of the DMV/NST complex following CT-HRP injection into the stomach wall and TMB processing. Labeled gastric motoneurons send dendrites primarily into the commissural and medial NST at intermediate and rostral levels. At rostral levels, dendrites exhibit extensive overlap with the gastric sensory terminal field in the subnucleus gelatinosus. Boxed area in $c$ is enlarged in Figure 2 for detail. $A P$, area postrema; $c c$, central canal; $D M V$, dorsal motor nucleus of the vagus; gel, subnucleus gelatinosus; $T r$, solitary tract; $4 t h \mathrm{~V}$, fourth ventricle. Scale bar, $0.5 \mathrm{~mm}$. followed by $200 \mathrm{ml}$ of dilute fixative $(0.1 \%$ paraformaldehyde, $0.5 \%$ TEM-grade glutaraldehyde in $0.1 \mathrm{M}$ sodium phosphate buffer at $\mathrm{pH} 7.4$ ), and $300 \mathrm{ml}$ of concentrated fixative ( 5 times the aldehyde concentration of the dilute fixative) in the same buffer. This was followed by infusion of $250 \mathrm{ml}$ of buffer to remove excess aldehydes. All solutions were infused at room temperature under controlled pressure with a peristaltic pump.

The brain stem was removed immediately following perfusion fixation. The rostrocaudal extent of the DMV/NST complex was serially sectioned in the coronal plane with a vibratome ( $50 \mu \mathrm{m} / \mathrm{section})$. Every third tissue section was reserved for light microscopic analysis, while the remaining sections were processed for EM analyses.

Light microscopy. Free-floating tissue sections were processed according to Mesulam's (1982) tetramethylbenzidine (TMB) procedure for light microscopic localization of CT-HRP (Shapiro and Miselis, 1985a, b; Rinaman and Miselis, 1987). Following the enzymatic reaction, sections were rinsed for $5 \mathrm{~min}$ in several changes of ice-cold sodium acetate buffer and then quickly mounted onto gelatin-coated slides and allowed to dry overnight at $4^{\circ} \mathrm{C}$. After rapid dehydration and coverslipping, sections were analyzed and photographed using bright- and dark-field illumination.

Electron microscopy. In 3 animals, half of the tissue sections intended for ultrastructural analysis of CT-HRP labeling were processed with TMB and the other half with diaminobenzidine (DAB). In 2 of these animals (one stomach-injected, one nerve-injected), the TMB protocol used was identical to that described above for light microscopy. In the third animal (stomach-injected), the pH of the TMB reaction was increased from 3.3 to 4.0 in an attempt to improve the structural preservation of the tissue, according to the procedure of Lemann and Saper (1985). The remaining tissue sections from those 3 animals and the complete set of tissue sections from 2 additional animals (one stomachinjected, one nerve-injected) were processed with DAB according to the procedure of Graham and Karnovsky (1966).

Following histochemical localization of CT-HRP, tissue sections were washed in several changes of buffer (either cold sodium acetate buffer or sodium phosphate buffer, following TMB or DAB reactions, respectively) and placed in a solution of $1 \%$ osmium tetroxide in $0.1 \mathrm{M}$ phosphate buffer ( $\mathrm{pH}$ 7.4). The osmium solution also contained $1.5 \%$ potassium ferricyanide, a treatment known to improve the structural preservation of aldehyde-fixed tissue (Langford and Coggeshall, 1980). Osmification was carried out for $1 \mathrm{hr}$ at room temperature with continuous agitation. Osmicated tissue was then washed in several changes of phosphate buffer, dehydrated in a graded ethanol series, and infiltrated and flat-embedded in Epon-Araldite plastic resin.

Analysis. Plastic flat-embedded sections were transilluminated and photographed to allow comparison of these sections with the alternate sections processed exclusively for light microscopy. This not only allowed evaluation of the intensity and extent of CT-HRP labeling that persisted following processing of the tissue for EM, but also ensured that subsequent ultrastructural observations were standardized by sampling the same rostrocaudal level of the DMV/NST in each animal. Each selected flat-embedded tissue section was trimmed down to a tissue block containing the right DMV/NST, medial to and including the tractus solitarius (see Fig. $4 a$ ). In every case, several series of ultrathin sections were collected on Formvar-coated slot grids, and a $0.5 \mu \mathrm{m}$ semithin section from the area immediately adjacent to each series of thin sections was collected and stained with $1 \%$ toluidine blue for light microscopic analysis. The relative distribution of reaction product was evaluated in these $0.5 \mu \mathrm{m}$ sections with a light microscope, and each section was photographed to provide landmarks for subsequent ultrastructural analysis.

A very systematic procedure was used in the EM analysis of each ultrathin section. Landmarks (blood vessels, the ventricular surface, and large neurons) identified in both the transilluminated $50 \mu \mathrm{m}$ sections and $0.5 \mu \mathrm{m}$ semithin sections were located at low magnification $(150 \times)$ on the electron microscope to identify the boundaries of the DMV and subnuclei of the NST. Following this orientation, sequential passes across the full extent of the thin section were made at a magnification of $20,000 \times$ (see Fig. $4 a$ for a schematic of this analysis) until the entire section had been analyzed. The identity of every CT-HRP-labeled profile encountered during these sequential passes was confirmed by examination at higher magnification, and photographed when deemed appropriate. This method of analysis ensured that each ultrathin section was examined in its entirety, and it also allowed precise determination of the location of every labeled profile as well as the relative distribution 


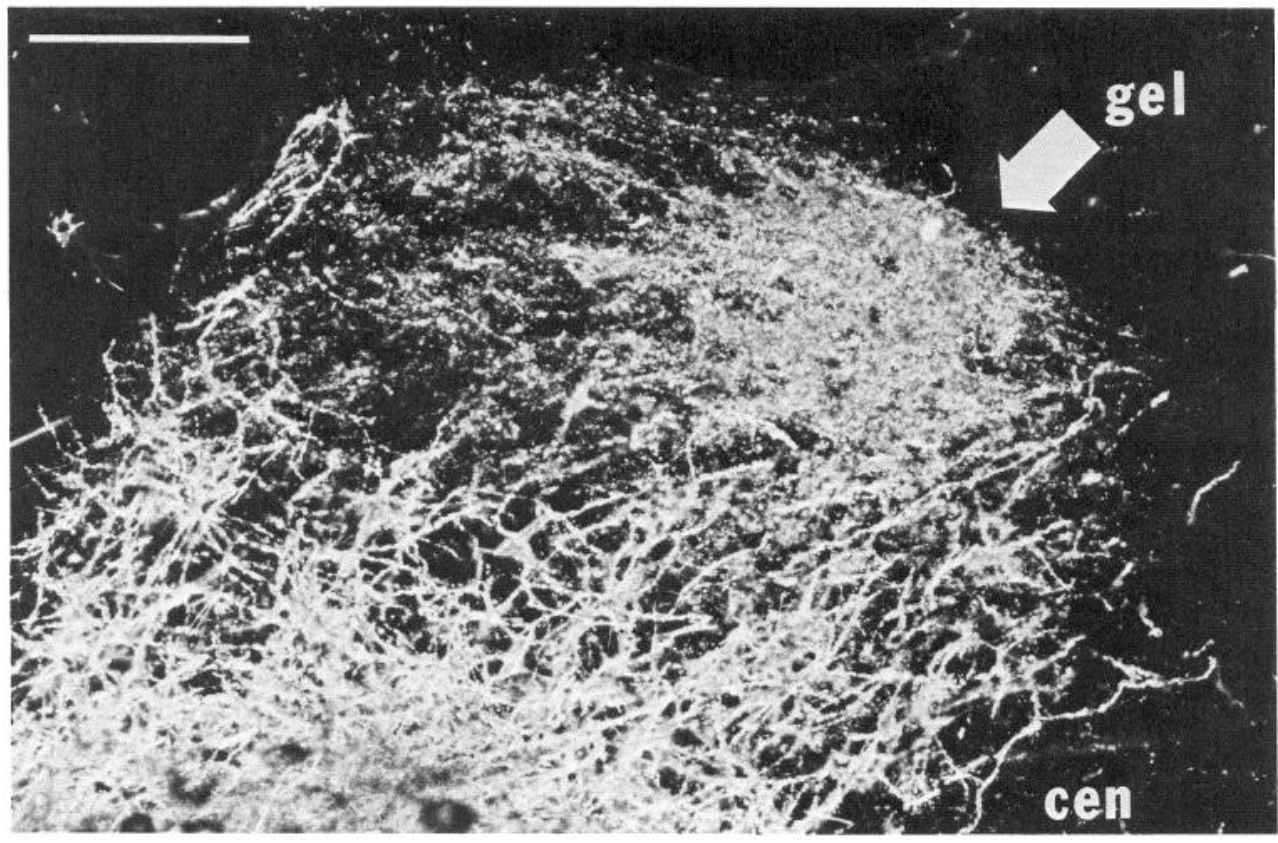

Figure 2. Enlargement of the boxed area in Figure $1 c$, photographed with dark-field illumination to show detail of dendritic-terminal overlap in the subnucleus gelatinosus following CTHRP injection into the stomach wall and TMB processing. cen, subnucleus centralis; gel, subnucleus gelatinosus. Scale bar, $0.5 \mathrm{~mm}$. of labeled profiles in the DMV and in the NST subnuclei. Ultrastructural analyses were conducted on uncounterstained material to facilitate the identification of the electron-dense reaction product.

\section{Results}

\section{Light microscopic analysis}

The labeling of neural profiles in the DMV/NST complex following CT-HRP injection into the dorsal and ventral musculature of the stomach is illustrated in Figures 1 and 2. Retrogradely labeled perikarya were located in the DMV bilaterally and gave rise to dendrites that extended well beyond the cytoarchitectural boundaries of the motor nucleus. Large numbers of labeled dendrites extended dorsally from the DMV into the medial NST, while smaller numbers of dendrites passed into the lateral NST, ventral to the subnucleus centralis (see Fig. $4 a$ for the locations of these NST subnuclei). At levels caudal to the obex, labeled dendrites crossed the midline to enter the contralateral DMV and medial NST (Fig. 1, $a, b$ ). Terminal labeling in stomach-injected animals was confined primarily to the dorsomedial NST and was particularly dense in the subnucleus gelatinosus at the level of the obex (Figs. 1c, 2). Consequently, tissue sections from this rostrocaudal level were selected for ultrastructural analysis as they contained the greatest overlap of labeled motoneuronal dendrites and sensory afferents. The subnucleus centralis, site of esophageal vagal afferent terminations (Altschuler et al., 1989), and areas of the NST lateral and dorsolateral to it contained little or no afferent labeling following stomach injection. The lack of labeling in esophageal regions of the NST served as an internal control indicating minimal leakage from the stomach injection sites. In addition, stomach injection did not result in labeling of motoneurons in the nucleus ambiguus, which provides motor innervation to the esophagus (Altschuler et al., 1989).

Following injection of the right cervical vagus nerve, the number of labeled DMV motoneurons was increased over that observed in stomach-injected animals. However, the distribution of labeled dendrites in the NST did not differ significantly (Fig. 3). Afferent labeling in the NST was much more extensive fol- lowing nerve injection (compare Figs. 3, $a-c$ and 1,a-c) than was observed following stomach injection. Specifically, the central, intermediate, interstitial, and ventrolateral subnuclei of the NST at the level of the obex (areas not labeled following stomach injection) contained moderate to dense afferent labeling following cervical nerve injection. The subnucleus gelatinosus of nerveinjected animals contained the same dense terminal labeling observed in stomach-injected animals.

Transillumination of TMB-reacted plastic-embedded tissue (Fig. $4 b$ ) revealed reaction product that was comparable in density and distribution to that observed in adjacent sections processed exclusively for light microscopy with TMB (Fig. 1c). In contrast, transillumination of plastic-embedded tissue that had been processed with $\mathrm{DAB}$ revealed only sparse reaction product in DMV perikarya and little or no visible afferent or dendritic labeling in the NST (Fig. 4c). Nevertheless, subsequent ultrastructural analysis of this DAB-reacted material revealed clearly labeled motoneurons, dendrites, and terminals, as described below.

\section{Electron microscopic analysis}

Briefly summarized, the present ultrastructural data demonstrate the existence of a central monosynaptic circuit between a portion of the sensory and motor components of the vagus nerve innervating the rat stomach. This circuit involves vagal gastric sensory terminals that synapse upon the distal dendrites of DMV motoneurons. At a level just rostral to the obex, these synaptic contacts occur almost exclusively in the subnucleus gelatinosus of the dorsomedial NST following either stomach or cervical nerve injection (Fig. 8).

The best ultrastructural preservation of tissue and specificity of labeling was achieved by DAB histochemistry. The electrondense $D A B$ reaction product was distinctive and easily identified despite its sparseness at the light-microscopic level. DAB reaction product was concentrated in multivesicular bodies, lipofuscin granules, and the smooth endoplasmic reticulum of labeled neurons and their dendrites (Figs. 5a;6,a-d;7a). DAB reaction product in labeled afferents was characteristically se- 


\section{Nerve}
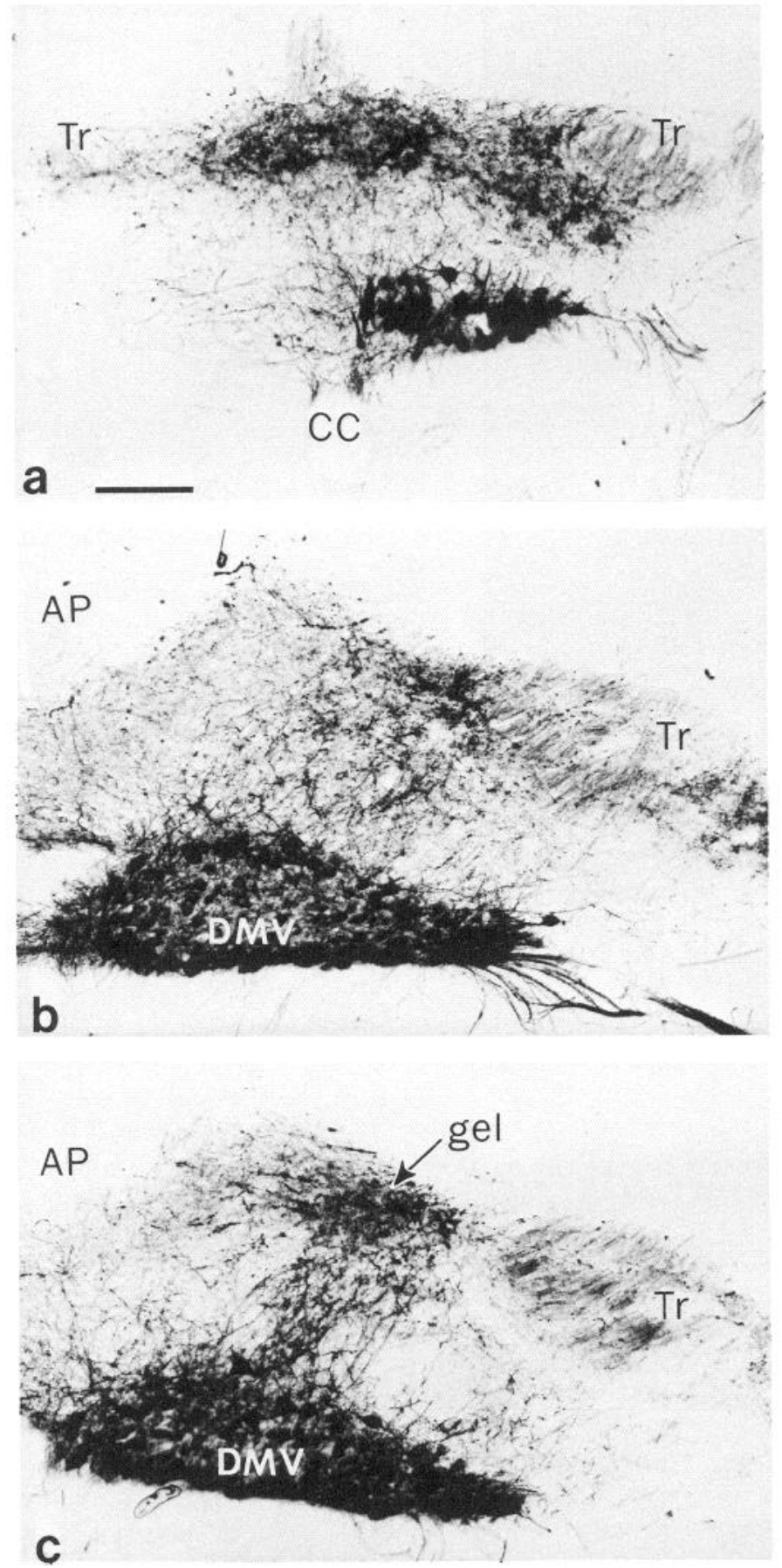

Figure 3. Light-field photomicrographs illustrating labeling of motoneurons and sensory afferents in $(a)$ caudal, $(b)$ intermediate, and $(c)$ rostral levels of the DMV/NST complex following CT-HRP injection into the right cervical vagus nerve and TMB processing. Note the expansion of the afferent field in the NST in these photomicrographs compared with those in Figure 1. In $c$, many labeled dendrites extend from the DMV to the densely labeled subnucleus gelatinosus of the NST. $A P$, area postrema; $c c$, central canal; $D M V$, dorsal motor nucleus of the vagus; gel, subnucleus gelatinosus; $T r$, solitary tract. Scale bar, $0.5 \mathrm{~mm}$. questered in small vesicles ranging in widest diameter from 20 to $40 \mathrm{~nm}$ (Figs. $5 b ; 6, a-d ; 7 b$ ). Ultrastructural analysis of tissue reacted with $\mathrm{TMB}$ at either $\mathrm{pH}$ revealed more extensive labeling of neuronal profiles, but the preservation of structure was inferior to that observed in DAB-reacted tissue (Fig. 5, $c, d$ ). The TMB reaction product consisted of electron-dense laminar arrays of crystalline particles that were larger than the $\mathrm{DAB}$ reaction product but that generally remained confined to neuronal profiles. The density of labeling within both DAB- and TMBlabeled profiles was directly related to the proximity of the thin section of the surface of the tissue block; i.e., thin sections close to the surface contained densely labeled profiles, while those from deeper in the block exhibited more discrete labeling. The integrity of membranes in TMB-reacted sections taken close to the surface of the tissue block was often disrupted, and organelles often exhibited artifacts related to the TMB processing. However, thin sections taken from deeper within the tissue block exhibited a marked improvement in structural preservation. Consequently, analysis of TMB-reacted tissue was restricted to ultrathin sections taken approximately $10 \mu \mathrm{m}$ into the tissue sample. These sections had the advantage of both improved structural morphology as well as more discrete TMB labeling, which was confined to labeled profiles and which did not obscure their identity during systematic analysis. Nevertheless, the structural preservation of DAB-processed tissue was always superior to TMB-processed tissue. We have therefore chosen to illustrate our current ultrastructural findings primarily with the DAB material, but it should be noted that these electron micrographs are representative of our observations of the pattern of labeling in both DAB- and TMB-processed tissue.

\section{Dorsal motor nucleus}

In all animals, labeled vagal motoneurons and labeled dendrites were observed in the DMV. Although the number of these labeled profiles was increased following nerve injection, no differences in the basic morphology of labeled neurons or dendrites were evident between stomach- and nerve-injected animals. Labeled dendrites in the DMV were heavily invested with synaptic inputs from a variety of morphologically distinct types of axon terminals. In only 4 instances were CT-HRP-labeled terminals found to be presynaptic to labeled dendrites in the DMV (Fig. $6 d$ ). In each of these instances, the labeled vagal afferents exhibited morphological characteristics identical to labeled afferents in the NST (see description below) and the postsynaptic labeled dendrite was small. It should be emphasized that the frequency with which such vagovagal contacts were observed within the confines of the DMV was extremely low, as only 4 definitive examples were observed in all of the experimental material analyzed. Labeled afferents were themselves quite rare in the DMV compared with their occurrence in the NST. Those present were of similar morphology and, excepting the 4 just mentioned, were always presynaptic to unlabeled dendrites when a synapse was observed.

\section{Nucleus of the solitary tract}

Labeling in the NST was restricted to dendrites, axons, and axon terminals. Labeled terminals in all areas of the NST (as well as those in the DMV) exhibited a homogeneously dense axoplasm containing dark mitochondria, lucent spherical vesicles averaging $40 \mathrm{~nm}$ in diameter, and occasional dense-core vesicles of larger caliber (Figs. 5, $b, d ; 6, a-d ; 7 b$ ). In both stomach- and nerve-injected animals, large labeled dendrites were 

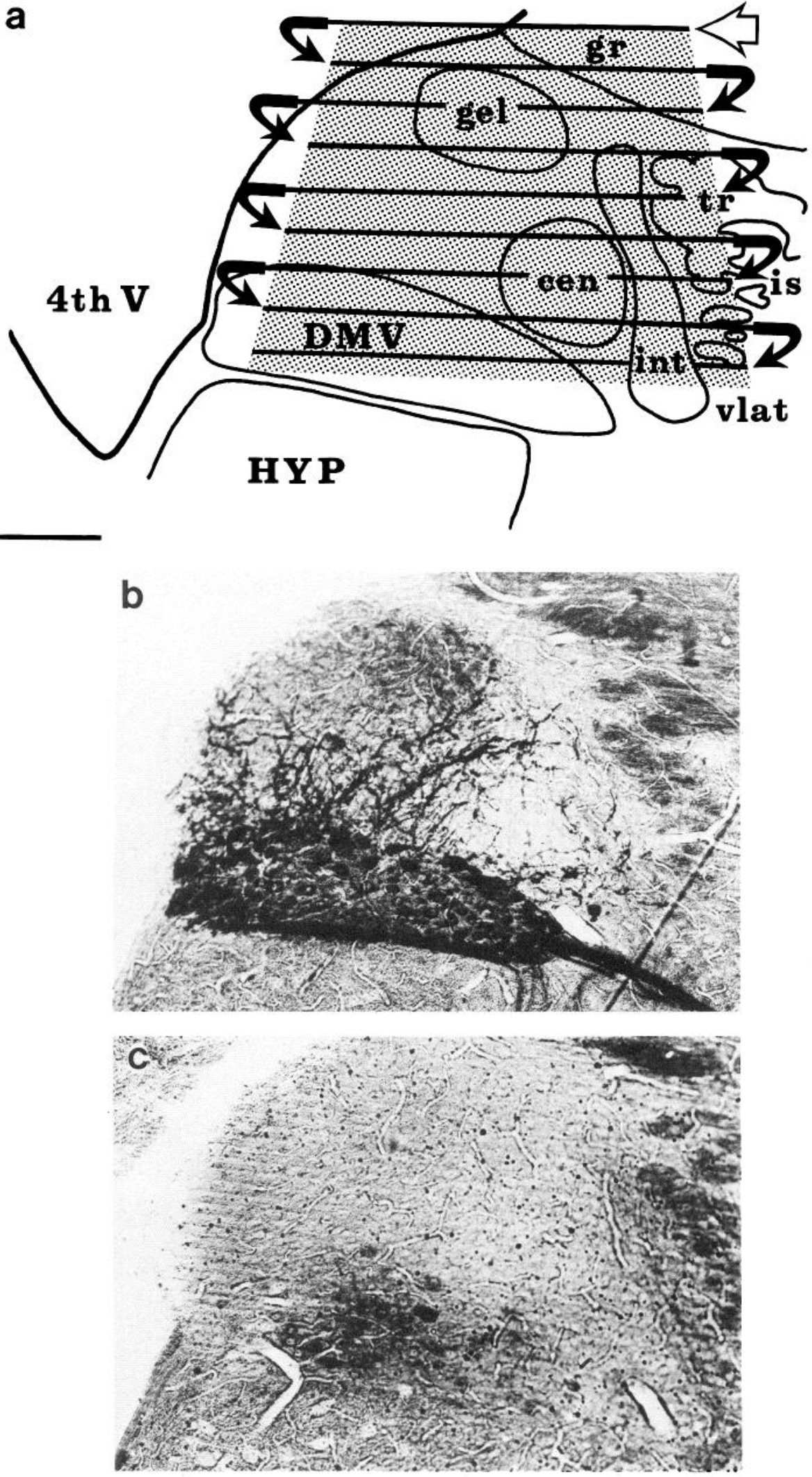

Figure 4. a, Line drawing of the right DMV/NST complex at the level of the obex. Stippled trapezoidal-shaped area indicates the region sampled for EM analysis of CT-HRP labeling. This figure is a tracing of the tissue section in $b$ and indicates the location of the NST subnuclei referred to in the present paper. Tracks represent progressive passes made over the entire thin section with the electron microscope at $20,000 \times$. These passes are drawn merely to indicate their direction; only a few representative passes are included in this schematic. cen, subnucleus centralis; $D M V$, dorsal motor nucleus of the vagus; gel, subnucleus gelatinosus; $g r$, gracile nucleus; $H Y P$, hypoglossal nucleus; int, intermediate subnucleus; is, interstitial subnucleus; $t r$, solitary tract; $v$ lat, ventrolateral subnucleus; $4 t h V$, fourth ventricle. Scale bar, $0.5 \mathrm{~mm}$. Panels $b$ and $c$ are photomicrographs of adjacent transilluminated plastic-embedded tissue sections from a stomach-injected animal, processed with either TMB $(b)$ or DAB $(c)$. TMB labeling is comparable in density and distribution to that seen in alternate tissue sections from the same animal processed exclusively for light microscopy (see Fig. 1c). In contrast, DAB labeling in the same fields is much sparser. commonly observed entering the NST from the underlying DMV. The widest diameter of these dendrites decreased with increasing distance from the DMV. In the medial NST ventral to the subnucleus gelatinosus, these labeled dendrites were frequently sectioned in the longitudinal plane and received numerous synaptic contacts from a variety of terminal types, always unlabeled (Fig. 7a). Labeled terminals were frequently observed in this region following stomach injection, although far more abun- 

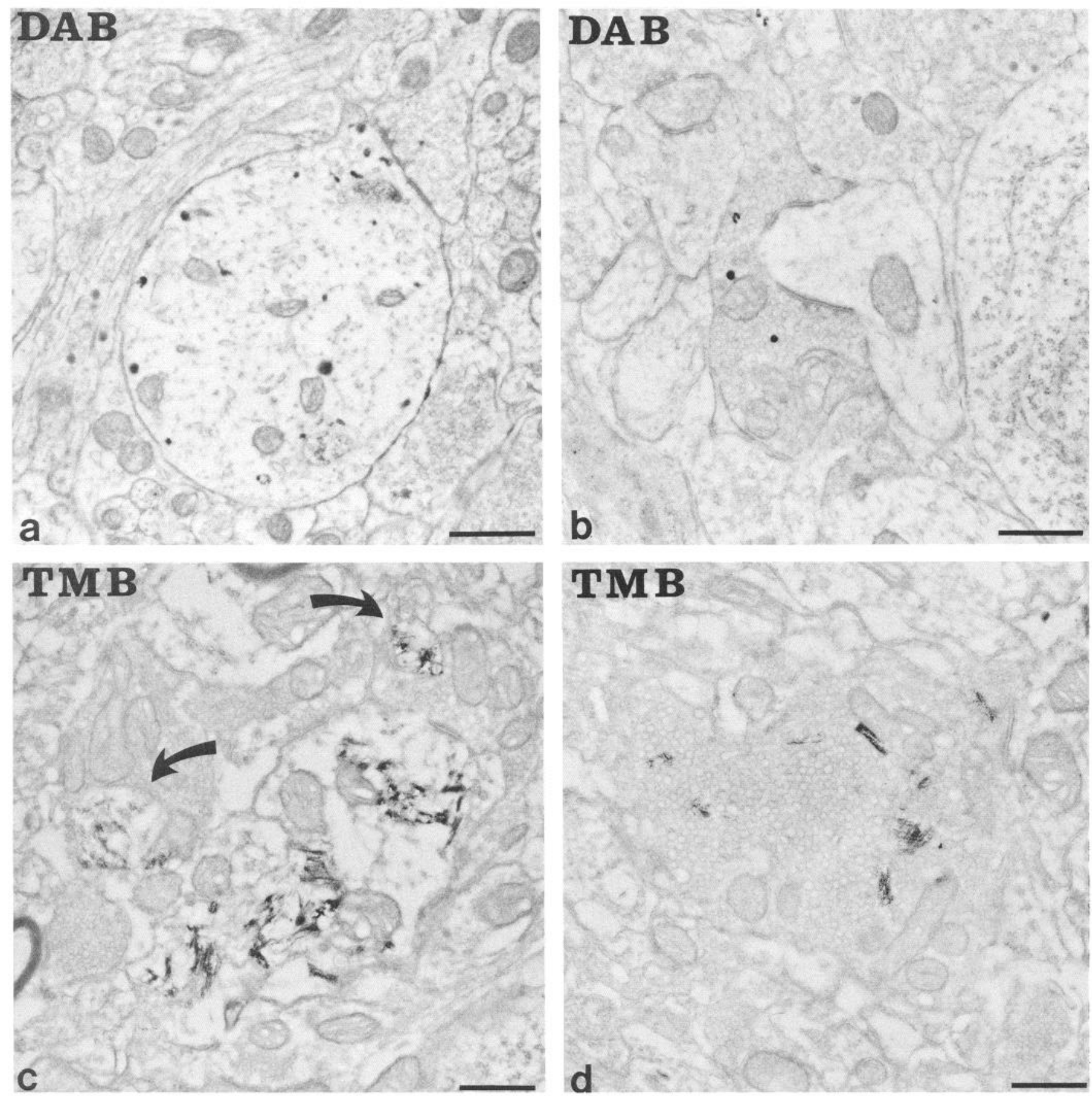

Figure 5. CT-HRP labeling of neuronal profiles in the subnucleus gelatinosus of the NST following DAB $(a$ and $b)$ or TMB $(c$ and $d)$ processing. $\mathrm{DAB}$ processing resulted in discrete, electron-dense labeling confined to cellular organelles in both dendrites $(a)$ and sensory afferents $(a$ and $b)$. In contrast, TMB labeling was characterized by dense crystalline accumulation that was not organelle-related but that generally remained confined to labeled dendrites $(c)$ and afferent terminals $(d)$. Note labeled dendritic spines indicated by arrows in $c$. DAB processing resulted in superior ultrastructural preservation of the tissue compared with TMB-processed material. Panels $a-c$ are from stomach-injected animals, while $d$ is from a nerve-injected animal. Scale bars, $0.5 \mu \mathrm{m}$.

dantly following nerve injection. These labeled terminals were always presynaptic to small, unlabeled dendrites and spines when synapses were observed (Fig. $7 b$ ).

Labeled dendrites were rarely encountered in the subnucleus centralis of the NST following stomach or nerve injection. Labeled afferents were virtually absent in this subnucleus following stomach injection but were prevalent following cervical nerve injection. These labeled terminals were always presynaptic to unlabeled profiles when a synapse was observed. Labeled dendrites were common in the region of the NST lateral to the subnucleus centralis and ventral to the tractus following both injection paradigms. Very few labeled terminals were observed in this region following stomach injection, and none were presynaptic to the labeled dendrites. Although labeled afferents 

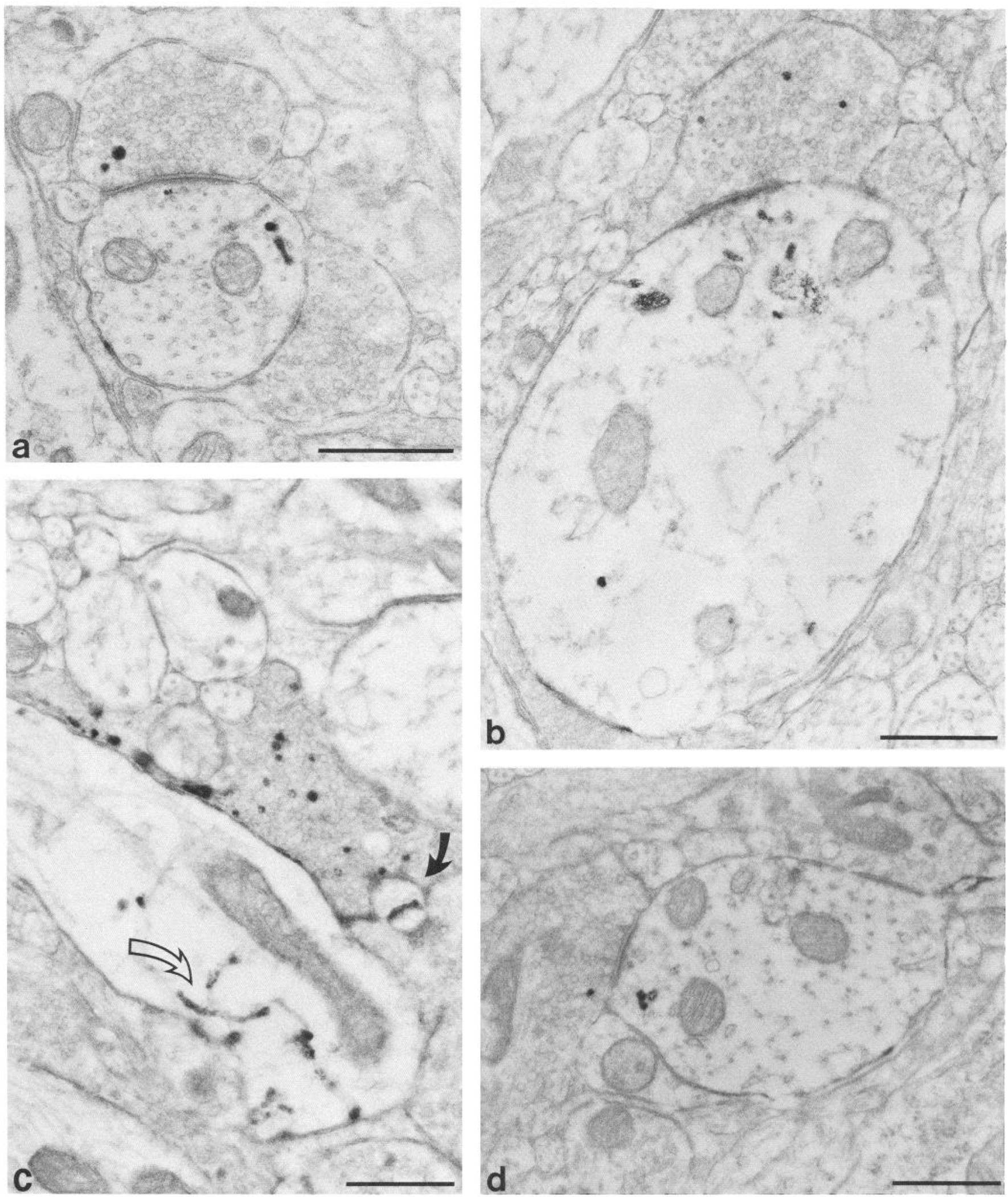

Figure 6. Electron micrographs from DAB-reacted tissue showing labeled vagovagal contacts following stomach injection. $a$, Labeled terminal (top) synapsing on a small labeled dendrite in the subnucleus gelatinosus. An unlabeled terminal (right) of the same morphology also synapses on the labeled dendrite. $b$, Labeled terminal (top) synapsing on a large labeled dendrite in the subnucleus gelatinosus. $c$, Labeled terminal (right) adjacent to a longitudinally sectioned labeled dendrite $(l e f t)$ in the subnucleus gelatinosus. The labeled terminal forms a synapse with a labeled dendritic spine (solid arrow). The labeled spine also receives a synapse from an unlabeled terminal of a different morphology. Note the association of DAB reaction product with the smooth endoplasmic reticulum (open arrow) in the labeled dendrite. $d$, One of the few examples of a labeled terminal (left) synapsing on a labeled dendrite (right) within the confines of the DMV. Scale bars, $0.5 \mu \mathrm{m}$. 


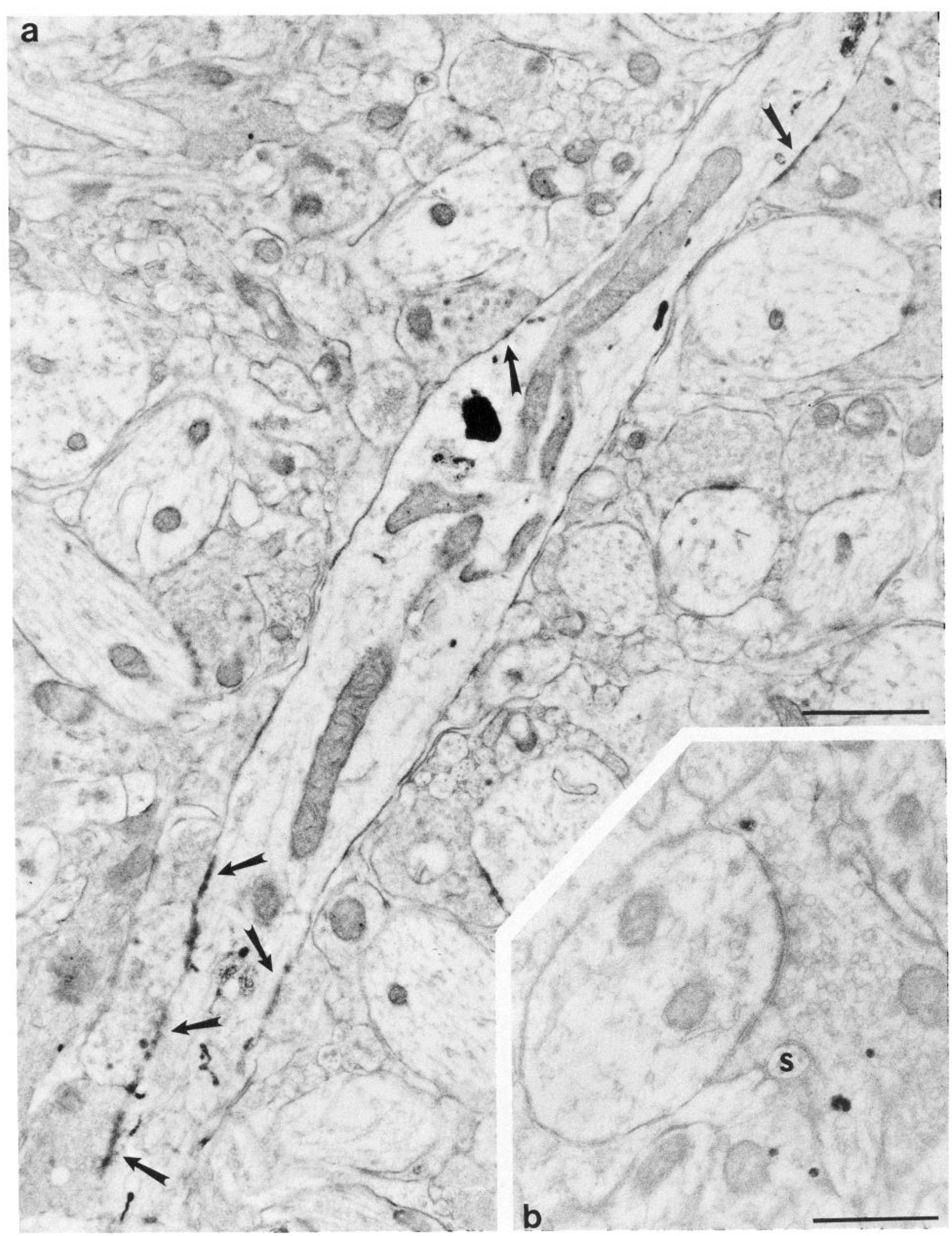

Figure 7. Electron micrographs of DAB labeling in the medial NST ventral to the subnucleus gelatinosus following stomach injection. $a$, A longitudinally sectioned labeled dendrite in the medial NST extending dorsally from the DMV in a direction towards the subnucleus gelatinosus. Along its course, this dendrite receives synapses from several terminals (arrows), none of which are labeled. Scale bar, $1.0 \mu \mathrm{m}$. The inset (b) at lower right shows a labeled terminal in this same region of the NST synapsing on a small, unlabeled dendrite and an unlabeled dendritic spine ( $s$ ). Scale bar, $0.5 \mu \mathrm{m}$. 
were prevalent here following nerve injection, they were in all cases presynaptic to unlabeled profiles when a synapse was observed.

Within the subnucleus gelatinosus of all animals, the majority of labeled dendrites were of intermediate to small caliber and were most often sectioned transversely or obliquely; longitudinally sectioned labeled dendrites were occasionally observed (see Fig. 6c). As in the other areas of the NST discussed above, these dendrites were postsynaptic to a variety of morphologically distinct axon terminals. However, in contrast to the other NST subfields, labeled sensory afferents in the subnucleus gelantinosus were frequently observed in synaptic contact with labeled dendrites and spines. Synaptic contacts between labeled terminal and labeled dendritic profiles were asymmetric and were characterized by presynaptic densities at sites of vesicle aggregation and a prominent postsynaptic density (Fig. 6). Labeled terminals were often located adjacent to neuronal perikarya in this region but were never observed in synaptic contact with them. The prevalence with which a vagal monosynaptic relationship was observed in the subnucleus gelatinosus of all experimental animals stands in sharp contrast to its almost complete absence in the DMV and in the other NST subdivisions examined. Eighty-five definitive examples of vagovagal contacts (including a clear, unambiguous synapse) were photographed in the course of this study, and only 4 (in the DMV) occurred outside of the subnucleus gelatinosus.

\section{Discussion}

Following CT-HRP injection into either the stomach wall or the cervical vagus nerve, ultrastructural examination of the DMV/NST complex at the rostrocaudal level of the obex revealed numerous synaptic contacts between labeled vagal sensory axon terminals and labeled vagal motoneuronal dendrites (Fig. 8). Although the physiological function of this monosynaptic vagovagal circuit is not known, it provides one anatomical explanation for certain electrophysiological data reported by other laboratories. In the in vitro slice preparation, stimulation of vagal sensory fibers leads to short-latency alterations in the electrical activity of vagal motoneurons (Yarom et al., 1985; Champagnat et al., 1986). Interestingly, one class of vagal motoneuronal response to afferent stimulation originates as postsynaptic unitary dendritic spikes in the distal dendrites of the motoneurons (Yarom et al., 1985), a phenomenon that is particularly relevant to our anatomical demonstration of vagovagal contacts between afferents and distal dendrites.

The present ultrastructural demonstration of labeled vagal motoneuronal dendrites and abundant vagovagal contacts in the NST was made possible by the use of a relatively new neural tracer, CT-HRP. To the best of our knowledge, the present investigation is the first to demonstrate that CT-HRP can be used in combination with either TMB or DAB histochemistry as a sensitive neural tracer at the EM level, revealing aspects of neural connectivity that have not been demonstrable using other tracers. Like free HRP and HRP conjugated to wheat germ agglutinin, CT-HRP is transported anterogradely and transganglionically to label axon terminals, as well as retrogradely, to label neuronal cell bodies (Wan et al., 1982a). In addition, CTHRP has the unique advantage of labeling the full dendritic tree of retrogradely filled neurons (Wan et al., 1982b). Previous control experiments have shown that the membrane-binding properties of CT make the CT-HRP conjugate extremely resistant
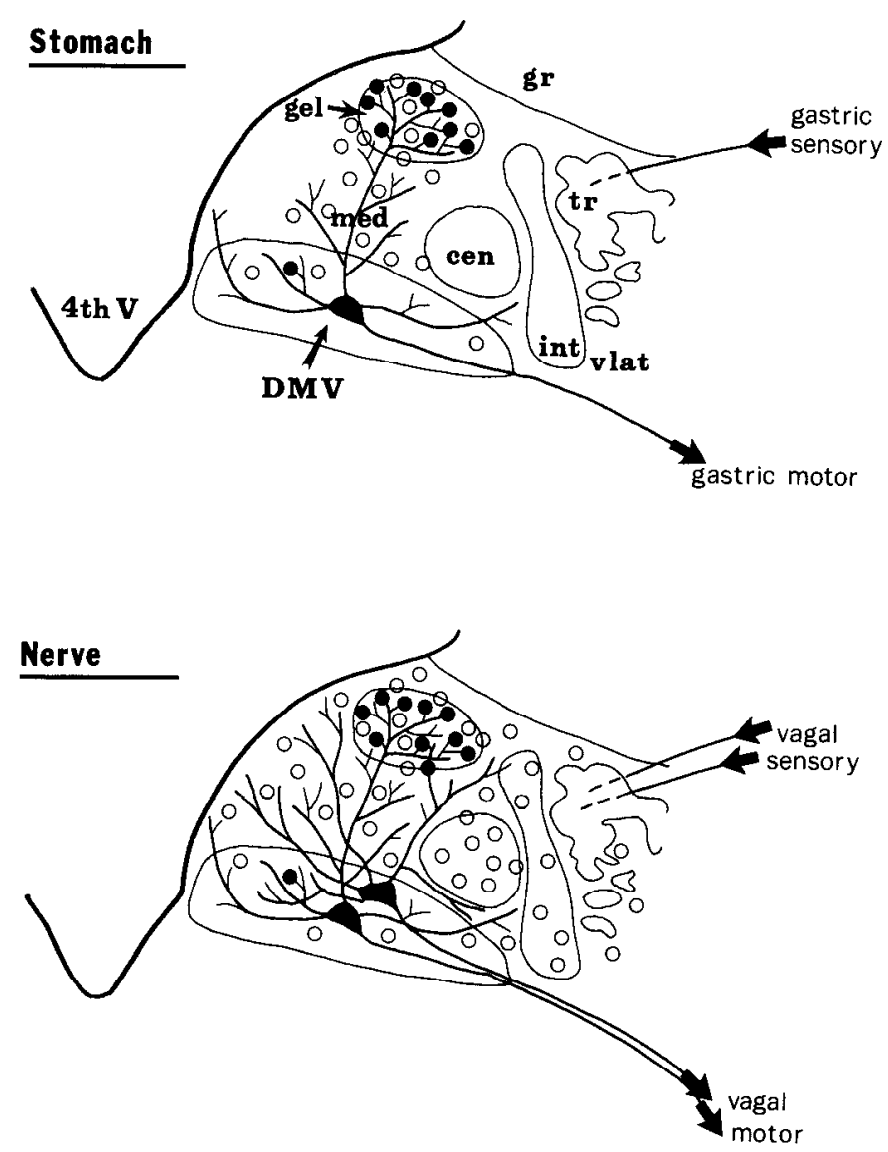

Figure 8. Top, Summary of the light and EM distribution of vagal sensory and motor labeling at the rostrocaudal level of the obex following stomach injection with CT-HRP. Gastric DMV neurons have spiny dendrites that branch within the DMV and the medial NST. The distal arborization of these dendrites is particularly prevalent in the subnucleus gelatinosus. A smaller number of dendrites course laterally beneath the subnucleus centralis but avoid entering this subnucleus. Gastric vagal sensory axons enter the tractus and terminate almost exclusively in the medial and gelatinosus subnuclei of the NST at this rostrocaudal level (circles). Open circles represent labeled gastric terminals that are not in synaptic contact with labeled motoneuronal dendrites, although they are often located near or adjacent to them. The gastric afferent field is concentrated in the subnucleus gelatinosus, and this subnucleus is the site for virtually all of the axodendritic vagovagal synaptic contacts observed in the present study (closed circles). Although a relatively small number of vagal afferents terminate in the DMV, a few of these terminals form synaptic contact there with gastric motoneuronal dendrites. Bottom, Following injection of the cervical vagus nerve, afferent labeling is found throughout the NST, and the number of labeled DMV motoneurons and dendrites is increased. However, the distribution of vagovagal contacts does not differ from stomach-injected animals. cen, subnucleus centralis; $D M V$, dorsal motor nucleus of the vagus; gel, subnucleus gelatinosus; $g r$, gracile nucleus; int, intermediate subnucleus; med, medial subnucleus; $t r$, tractus; $4 t h V$, fourth ventricle.

to diffusion away from peripheral injection sites even with postinjection survival times of several days, thereby limiting inappropriate neural labeling due to tracer spread (Rinaman and Miselis, 1987). For the stomach-injected animals in the present study, the absence of afferent labeling in the subnucleus centralis of the NST, site of esophageal afferent termination (Altschuler et al., 1989), serves as an internal control indicating that tracer leakage away from the injection sites in the stomach wall was minimal. 
Labeled sensory afferents were rarely encountered in the DMV, supporting previous reports in rat, cat, and monkey (Cottle, 1964; Norgren, 1978; Leslie et al., 1982a; McLean and Hopkins, 1985; Neuhuber and Sandoz, 1986). In Neuhuber and Sandoz's (1986) study, injection of free HRP into the rat cervical vagus nerve resulted predictably in sparse terminal labeling in the DMV; however, a few examples of labeled terminals in synaptic contact with labcled dendrites in the DMV were found. Our observations are consistent with theirs, as only 4 vagovagal contacts were observed in the DMV in all of the tissue examined in the current study. In contrast, however, a total of 81 definitive examples of vagovagal axodendritic synaptic contacts were found in the subnucleus gelatinosus in the dorsomedial NST in thin sections examined from both TMB- and DAB-reacted tissue from all 5 animals. The ease with which vagovagal contacts could be demonstrated suggests that they are abundant in this subnucleus. Although labeled dendritic and afferent profiles were present in varying degrees in other areas of the NST at this rostrocaudal level, thorough examination revealed that labeled afferents and dendrites never formed synaptic contacts with one another in these other NST subregions in either stomach- or nerve-injected animals.

In TMB-reacted tissue, the size of the reaction product resulted in more completc filling of labeled profiles than did the much smaller DAB reaction product. Consequently, sections through TMB-labeled profiles were more likely to contain electron-dense reaction product, and so TMB-labeled profiles presented themselves more frequently in a given thin section than did DAB-labeled profiles. However, ultrastructural preservation in DAB-reacted tissue was superior to that obtained with TMB histochemistry, and so clear demonstrations of synaptic specializations between labeled profiles were more commonly observed in DAB-reacted material. The discrete size of the DAB reaction product leads one to believe that the number of $D A B$ labeled afferents, dendrites, and vagovagal contacts observed in the present study is a potentially large underestimation of the actual number. This underestimation, however, should not affect the relative distribution of labeled profiles and vagovagal contacts observed.

Injection of the right cervical vagus nerve resulted in neither an obviously greater nor a more widespread occurrence of labeled vagovagal contacts than stomach injection. The most extensive overlap of vagal motoneuronal dendrites and terminals occurred in the gastric-related subnucleus gelatinosus of the dorsomedial NST, both when the stomach was injected with tracer as well as when the cervical vagus nerve was injected. The morphology of labeled afferents was quite consistent in all specimens examined and corresponds with previous reports in cat and rat (Leslie et al., 1982b; Neuhuber and Sandoz, 1986). Afferent labeling was more widely distributed in the NST following cervical nerve injection than following stomach injection, as expected given the more restricted access to vagal sensory fibers afforded by stomach injection. However, although the number of labeled DMV neurons and dendrites was higher following nerve injection than following stomach injection, the distribution of labeled dendrites in the NST was quite similar in all animals. At the level of the obex, the majority of labeled motoneuronal dendrites following either stomach or whole nerve injection were located in the medial NST, although in both injection paradigms a smaller number of labeled dendrites extended into the lateral NST beneath the tractus.

The functional significance of a central gastric vagovagal cir- cuit is open to speculation. The monosynaptic contact could provide a short-latency neuromodulation whereby gastric afferent information is relayed directly to the appropriate vagal effectors, as would be consistent with many classically described cephalic vagovagal reflexes (Harper et al., 1959; Blackshaw et al., 1987). Vagal motor function is based on central relay and reflex systems, which in large part depend on afferent sensory input (IIarper et al., 1959; Burnstock et al., 1979; Novin et al., 1981; Powley and Laughton, 1981; Blackshaw et al., 1987). Although the enteric nervous system of the gut possesses its own local reflex circuitry that can act independently of central innervation (Cannon, 1906; Burnstock et al., 1979; Gonella et al., 1987), a central monosynaptic gastric circuit could provide finely tuned feedback control over these enterically based reflexes by integrating and modulating other central inputs to vagal motoneurons (Delbro and Gustafsson, 1986; Grundy et al., 1986), suggesting the importance of constant and immediate vagal afferent feedback in controlling efferent output at the level of the motoneuron itself.

Based on electrophysiological results, it has been proposed that mechanosensitive vagal afferent input to the brain stem is more important in the genesis of gastrointestinal vagal reflexes than is chemosensitive afferent input (Blackshaw et al., 1987). This raises the interesting possibility that the terminals involved in vagovagal contacts might transmit gastric mechanoreceptive information, while vagal gastric afferents that are not part of this monosynaptic circuit might relay chemoreceptive information. A multitude of possible roles for monosynaptic central contacts involving mechanoreceptors exist. One example may lie in the regulation of gastric reflux. Emesis requires fine-grain temporal and spatial resolution in its control; since rats do not normally vomit, the monosynaptic circuit may have a role in preventing vomiting. In species that do predictably vomit (i.e., carnivores and ruminants), this reflex may differ in organization or chemistry or be influenced differently by other central inputs. Other possible roles for this monosynaptic circuit include receptive relaxation of the stomach in the presence of food, gastric secretion in response to stretch of the gastric wall, the timing of gastric contractions, or the maintenance of gastric muscular "tone." Species differences in the physiology of many vagal reflexes may well correlate with species differences in the central synaptology or neurochemistry of vagal afferents and efferents.

Apart from gastrointestinal physiology, the innervation of vagal motoneurons by vagal sensory neurons may help explain the especially poor survival of vagal motoneurons following peripheral nerve damage, as compared with neurons in other brain-stem motor nuclei (Lewis et al., 1972; Aldskogius et al., 1980). It is not known why the majority of vagal motoneurons respond to peripheral axotomy degeneratively, because their axons initially undergo normal regenerative growth (Guth and Jacobson, 1966). However, as the vagus nerve is a mixed nerve, peripheral damage necessarily affects both motor and sensory components. The current results indicate that a direct source of afferent information and/or chemicals normally available to certain vagal motoneurons would be lost following vagotomy. This loss would certainly be irreversible following vagotomy above the vagal sensory ganglion (supranodose vagotomy), and as transganglionic degeneration of the central vagal sensory terminals has been reported following infranodose vagotomy (Majumdar et al., 1983; Ling et al., 1986), this loss may be permanent following damage to the nerve below its sensory ganglion as well. Long-term anterograde effects of neurons on the cells 
they innervate are crucial in maintaining the normal properties and in some cases even the survival of those cells (Cook et al., 1951; Kuffler et al., 1971; Knyihar-Csillik and Csillik, 1981; Saji and Reis, 1987; Benshalom and White, 1988). More specifically, physiological studies have shown that intact vagal sensory input to the NST is necessary for the normal ongoing activity of gastric motoneurons (Barber and Burks, 1983). Partial denervation of DMV neurons might therefore be a factor in their necrobiotic retrograde response to peripheral axotomy. As the severity of this response varies between mammalian species (Lewis et al., 1972; Navaratnam and Lewis, 1975; Aldskogius, 1978; Ling et al., 1986; Laiwand et al., 1987), it would be intriguing to discover whether such differences correlate with vagovagal synaptology in the DMV/NST complex.

In summary, vagovagal axodendritic synapses occur in abundance in the NST of the rat. At the rostrocaudal level just anterior to the obex, these vagovagal contacts are limited almost exclusively to the gastrointestinal-related subnucleus gelatinosus of the dorsomedial NST (Fig. 8). This suggests that the monosynaptic vagovagal circuit may be exclusively a gastric phenomenon, although the possible presence and distribution of such contacts in areas of the vagal complex at other rostrocaudal levels cannot be excluded.

\section{References}

Aldskogius, H. (1978) Fine structural changes in nerve cell bodies of the adult rabbit dorsal motor vagal nucleus during axon reaction. Neuropathol. Appl. Neurobiol. 4: 323-341.

Aldskogius, H., K. D. Barron, and R. Regal (1980) Axon reaction in dorsal motor vagal and hypoglossal neurons of the adult rat. Light microscopy and RNA-cytochemistry. J. Comp. Neurol. 193: 165177

Altschuler, S. A., X. Bao, D. Bieger, D. A. Hopkins, and R. R. Miselis (1989) Viscerotopic representation of the upper alimentary tract in the rat: Sensory ganglia and the nuclei of the solitary and spinal trigeminal tracts. J. Comp. Neurol. (in press).

Barber, W. D., and T. F. Burks (1983) Brain stem response to phasic gastric distension. Am. J. Physiol. 8: G242-G248.

Benshalom, G., and E. L. White (1988) Dendritic spines are susceptible to structural alterations induced by degeneration of their presynaptic afferents. Brain Res. 443: 377-382.

Blackshaw, L. A., D. Grundy, and T. Scratcherd (1987) Involvement of gastrointestinal mechano- and intestinal chemoreceptors in vagal reflexes: An electrophysiological study. J. Auton. Nerv. Syst. 18: 225234.

Burnstock, G., T. Hökfelt, M. D. Gershon, L. L. Iversen, H. W. Kosterlitz, and J. H. Szurszewski (1979) Non-adrenergic, non-cholinergic autonomic neurotransmission mechanisms. Neurosci. Res. Prog. 17(3): 392-401.

Cannon, W. B. (1906) The motor activities of the stomach and small intestine after splanchnic and vagus section. Am. J. Physiol 17: 429444.

Champagnat, J., M. Denavit-Saubie, K. Grant, and K. F. Shen (1986) Organization of synaptic transmission in the mammalian solitary complex, studied in vitro. J. Physiol. (Lond.) 381: 551-573.

Cook, W. H., J. H. Walker, and M. L. Barr (1951) A cytological study of transneuronal atrophy in the cat and rabbit. J. Comp. Neurol. 94: 267-291.

Cottle, M. (1964) Degeneration studies of primary afferents of the IXth and Xth cranial nerves in the cat. J. Comp. Neurol. 122: 329 343.

Delbro, D., and B. Gustafsson (1986) Vagally and vago-vagally induced non-adrenergic, non-cholinergic relaxations of the feline jejunum. Acta Physiol. Scand. 128: 125-126.

Ewart, W. R., and D. L. Wingate (1983) Central representation and opioid modulation of gastric mechanoreceptor activity in the rat. Am. J. Physiol. 244: G27-G32.

Gonella, J., M. Bouvier, and F. Blanquet (1987) Extrinsic nervous control of motility of small and large intestines and related sphincters. Physiol. Rev. 67: 902-961.
Graham, R. C., and M. J. Karnovsky (1966) The early stages of absorption of injected horseradish peroxidase in the peripheral tubules of mouse kidney: Ultrastructural cytochemistry by a new technique. J. Histochcm. Cytochcm. 14: 291-302.

Grundy, D., D. Hutson, and T. Scratcherd (1986) A permissive role for the vagus nerves in the genesis of antro-antral reflexes in the anaesthetized ferret. J. Physiol. (Lond.) 381: 377-384.

Guth, L., and S. Jacobson (1966) The rate of regeneration of the vagus nerve of the cat. Exp. Neurol. 14: 439-447.

Harper, A. A., C. Kidd, and T. Scratcherd (1959) Vago-vagal reflex effects on gastric and pancreatic secretion and gastrointestinal motility. J. Physiol. (Lond.) 148: 417-436.

Knyihar-Csillik, E., and B. Csillik (1981) Selective 'labeling' by transsynaptic degeneration of substantia gelatinosal cells: An attempt to decipher intrinsic wiring in the rolando substance of primates. Neurosci. Lett. 23: 131-136.

Kuffler, S. W., M. J. Dennis, and A. J. Harris (1971) The development of chemosensitivity in extrasynaptic areas of the neuronal surface after denervation of parasympathetic ganglion cells in the heart of the frog. Proc. R. Soc. London [Biol.] 177: 555-563.

Laiwand, R., R. Werman, and Y. Yarom (1987) Time course and distribution of motoneuronal loss in the dorsal motor vagal nucleus of guinea pig after cervical vagotomy. J. Comp. Neurol. 256: 527537 .

Langford, L. A., and R. E. Coggeshall (1980) The use of potassium ferricyanide in neural fixation. Anat. Rec. 197: 297-303.

Lemann, W., and C. B. Saper (1985) Stabilization of TMB reaction product for electron microscopic retrograde and anterograde fiber tracing. Brain Res. Bull. 14:277-281.

Leslie, R. A., D. G. Gwyn, and D. A. Hopkins (1982a) The central distribution of the cervical vagus nerve and gastric afferent and efferent projections in the rat. Brain Res. 8: 37-43.

Leslie, R. A., D. G. Gwyn, and D. A. Hopkins (1982b) The ultrastructure of the subnucleus gelatinosus of the nucleus of the tractus solitarius in the cat. J. Comp. Neurol. 206: 109-118.

Lewis, P. R., P. Blundell Jones, S. M. Breathnach, and V. Navaratnam (1972) Regenerative capacity of visceral preganglionic neurons. $\mathrm{Na}$ ture [New Biol.] 236: 181-182.

Ling, E. A., W. C. Wong, T. Y. Yick, and S. K. Leong (1986) Ultrastructural changes in the dorsal motor nucleus of monkey following bilateral cervical vagotomy. J. Neurocytol. 15: 1-15.

Majumdar, S., E. Mills, and P. G. Smith (1983) Degenerative and regenerative changes in central projections of glossopharyngeal and vagal sensory neurons after peripheral axotomy in cats: A structural basis for central reorganization of arterial chemoreflex pathways. Neuroscience 10: 841-849.

McLean, J. H., and D. A. Hopkins (1985) Ultrastructure of the dorsal motor nucleus of the vagus nerve in monkey with a comparison of synaptology in monkey and cat. J. Comp. Neurol. 231: 162-174.

Mesulam, M.-M. (1982) Principles of horseradish peroxidase neurohistochemistry and their applications for tracing neural pathwaysaxonal transport, enzyme histochemistry, and light microscopic analysis. In Tracing Neural Connections With Horseradish Peroxidase, M.-M. Mesulam, ed., pp. 1-151, Wiley, Chichester, UK.

Navaratnam, V., and P. R. Lewis (1975) Effects of vagotomy on the distribution of cholinesterases in the cat medulla oblongata. Brain Res. 100: 599-613.

Neuhuber, W. L., and P. A. Sandoz (1986) Vagal primary afferent terminals in the dorsal motor nucleus of the rat: Are they making monosynaptic contacts on preganglionic efferent neurons? Neurosci. Lett. 69: 126-130.

Norgren, R. (1978) Projections from the nucleus of the solitary tract in the rat. Neuroscience 3:207-218.

Novin, D., R. C. Rogers, and G. Hermann (1981) Visceral afferent and efferent connections in the brain. Diabetologia 20: 331-336.

Powley, T. L., and W. Laughton (1981) Neural pathways involved in the hypothalamic integration of autonomic responses. Diabetologia 20: 378-387.

Rinaman, L., and R. R. Miselis (1987) The organization of vagal innervation of rat pancreas using cholera toxin-horseradish peroxidase conjugate. J. Autonom. Nerv. Syst. 21: 109-125.

Saji, M., and D. J. Reis (1987) Delayed transneuronal death of substantia nigra neurons prevented by $\gamma$-aminobutyric acid agonist. Science 235: 66-68.

Sawchenko, P. E., E. T. Cunningham, Jr., and M. C. Levin (1987) 
Anatomic and biochemical specificity in central autonomic pathways. In Organization of the Autonomic Nervous System: Central and Peripheral Mechanisms, J. Ciriello, F. R. Calaresu, L. P. Renaud, and C. Polosa, eds., pp. 267-281, Liss, New York.

Shapiro, R. E., and R. R. Miselis (1985a) The central organization of the vagus nerve innervating the stomach of the rat. J. Comp. Neurol. 238: 473-488.

Shapiro, R. E., and R. R. Miselis (1985b) The central neural connections of the area postrema of the rat. J. Comp. Neurol. 234: 344-364. Szentagothai, J. (1948) Anatomical considerations of monosynaptic reflex arcs. J. Neurophysiol. 11: 445-454.
Wan, X. S. T., J. Q. Trojanowski, and J. O. Gonatas (1982a) Cholera toxin and wheat germ agglutinin conjugates as neuroanatomical probes: Their uptake and clearance, transganglionic, and retrograde transport and sensitivity. Brain Res. 243: 215-224.

Wan, X. S. T., J. Q. Trojanowski, J. O. Gonatas, and C. N. Lui (1982b) Cytoarchitecture of the extranuclear and commissural dendrites of hypoglossal nucleus neurons as revealed by conjugates of horseradish peroxidase with cholera toxin. Exp. Neurol. 78: 167-175.

Yarom, Y., M. Sugimori, and R. Llinás (1985) Ionic currents and firing patterns of mammalian vagal motoneurons in vitro. Neuroscience 16: 719-737. 